Artículo Original

\title{
Factores de riesgo cardiovascular en una población joven de Coronel Oviedo, Paraguay
}

\section{Cardiovascular risk factors in a young population of Coronel Oviedo, Paraguay}

Autores: Marcial Carlos González Galeano ${ }^{1}$, Magali Aguilera Sánchez ${ }^{2}$, Abel Benítez Valdez ${ }^{2}$, Noemí Biagioni Brítez $^{2}$, Lorena Cabañas Huber ${ }^{2}$, María Casco Larroza ${ }^{2}$, Fernando Espínola Chamorro² ${ }^{2}$ Clarisa Genes Rolón ${ }^{2}$, Elsa Godoy Notario ${ }^{2}$, Katia López ${ }^{2}$

\section{Resumen}

Introducción: Las enfermedades cardiovasculares constituyen la primera causa de mortalidad a nivel mundial. Las causas implicadas en las mismas están bien definidas y son los denominados factores de riesgo cardiovascular. Es de utilidad conocer la frecuencia de estos factores en la población adulta joven, de modo a tomar medidas preventivas.

Objetivo: determinar la prevalencia de hipertensión arterial, diabetes mellitus, sobrepeso, obesidad y tabaquismo en población joven del área urbana de la ciudad de Coronel Oviedo, Paraguay.

Material y método: se realizó un estudio, observacional, descriptivo de corte transversal, entre los meses de junio a agosto del 2012. Se incluyó a 139 sujetos entre 20 y 40 años, del área urbana de la ciudad de Coronel Oviedo, seleccionados mediante muestreo aleatorio simple, utilizando las recomendaciones del método progresivo paso a paso (steps) de vigilancia de factores de riesgo, de la Organización Mundial de la Salud y la Organización Panamericana de la Salud.

Resultados: la edad media fue 28,8 años, la distribución según sexo fue de $63 \%$ femenino y $37 \%$ masculino. El estudio demostró que 21\% padece de hipertensión arterial, 8\% diabetes mellitus, 36\% sobrepeso, 14\% obesidad y 18\% tabaquismo. El 61\% de los encuestados presentaba factores de riesgo cardiovascular, distribuidos de la siguiente manera: 1 factor de riesgo 37\%, 2 factores de riesgo 19\% y 3 o más factores de riesgo $6 \%$.

Conclusiones: los factores de riesgo cardiovascular más frecuentes fueron el sobrepeso, hipertensión arterial y tabaquismo.

\footnotetext{
${ }^{1}$ Especialista en Medicina Interna. Hospital Regional Dr. José A. Samudio. Facultad de Ciencias Médicas, Universidad Nacional de Caaguazú. Coronel Oviedo, Paraguay.

${ }^{2}$ Estudiantes de Cátedra de Clínica Médica. Facultad de Ciencias Médicas. Universidad Nacional de Caaguazú. Coronel Oviedo, Paraguay.

Autor correspondiente

Dr. Marcial Carlos César González Galeano

Médico de Planta e Instructor de Semiología Médica

Facultad de Ciencias Médicas, Universidad Nacional de Caaguazú.

Dirección: Wilfrido Martínez s/№ Km. 130. Coronel Oviedo, Paraguay

Tel: (595) 21205703

Correo electrónico: marcial2ccgg@hotmail.com
} 
Palabras claves: factores de riesgo cardiovascular, adulto joven, hipertensión arterial, obesidad, diabetes mellitus.

\begin{abstract}
Introduction: Cardiovascular diseases are the first cause of mortality worldwide. The implicated causes of them are well defined and are called cardiovascular risk factors. It is useful to know the frequency of these factors in the young adult population in order to take preventive measures.
\end{abstract}

Objective: To determine the prevalence of arterial hypertension, diabetes mellitus, overweight, obesity and smoking in the young population of the urban area of Coronel Oviedo, Paraguay.

Material and methods: A cross-sectional, descriptive observational study was carried out from June to August, 2012. One hundred thirty nine subjects, who were between 20 and 40 years of age from the urban area of Coronel Oviedo, were included. They were selected by simple random sampling using the recommendations of the stepwise approach to Surveillance (STEPS) for risk factors of the World Health Organization and the Pan American Health Organization.

Results: Mean age was 28.8 years, distribution by sex was $63 \%$ women and $37 \%$ men. The study showed that $21 \%$ suffered arterial hypertension, $8 \%$ diabetes mellitus, $36 \%$ overweight, $14 \%$ obesity and $18 \%$ smoking. Sixty one percent presented cardiovascular risk factors distributed as follows: one risk factor $37 \%$, two risk factors $19 \%$ and three or more risk factors $6 \%$.

Conclusions: The most frequent cardiovascular risk factors were overweight, arterial hypertension and smoking.

Keywords: cardiovascular risk factors, adult, arterial hypertension, obesity, diabetes mellitus.

\title{
Introducción
}

Las enfermedades crónicas no transmisibles son responsables cada año de la muerte de más de 36 millones de personas aproximadamente en todo el mundo, el 80\% de las defunciones por enfermedades no transmisibles se producen en los países de ingresos bajos y medios, más de 9 millones de las muertes atribuidas a las enfermedades no transmisibles se producen en personas menores de 60 años de edad, el $90 \%$ de estas muertes prematuras ocurren en países de ingresos bajos y medianos, las enfermedades cardiovasculares constituyen la mayoría de las defunciones, aproximadamente 17 millones cada año. En términos de muertes atribuibles, el principal factor de riesgo cardiovascular es la hipertensión arterial, a la que se le atribuye 13\% de las defunciones a nivel mundial, seguido por el consumo de tabaco (9\%), el aumento de la glucosa sanguínea (6\%), la inactividad física (6\%), el sobrepeso y la obesidad (5\%). Estos datos afirman que las enfermedades cardiovasculares constituyen la primera causa de mortalidad a nivel mundial. ${ }^{1}$

Las causas de las enfermedades cardiovasculares están bien definidas, son bien conocidas, y los desencadenantes más importantes son los denominados factores de riesgo cardiovascular. Cuando se hace mención al riesgo cardiovascular, representa la posibilidad de daño; en las diferentes estructuras y funciones del individuo; es más, si persisten en el tiempo estos factores de riesgo, hay una probabilidad acentuada de padecer enfermedad cardiovascular.

Los diversos factores de riesgo cardiovascular presentan características particulares. Algunos son denominados factores no modificables como el sexo, la raza, los antecedentes familiares de enfermedad cardiovascular y la edad avanzada. Existe otro grupo de factores de riesgo denominado modificables: el 
sobrepeso, la obesidad, el sedentarismo, las dislipidemias, así como el tabaquismo. Este último grupo se halla en relación directa con los estilos de vida de las personas. Se debe destacar a la hipertensión arterial y la diabetes mellitus porque son de alta prevalencia e incidencia.

Para evaluar el nivel de riesgo cardiovascular de la población latinoamericana, se diseñó un estudio en siete ciudades de la región, a fin de realizar un muestreo poblacional homogéneo con metodología única, exámenes de laboratorio y físicos estandarizados y controlados para obtener así datos epidemiológicos confiables. El estudio C.A.R.M.E.L.A. (Cardiovascular Risk Factor Multiple Evaluation in Latin America) evaluó 11.550 sujetos de la población general, entre 25-64 años de edad, en siete ciudades: Barquisimeto en Venezuela, Bogotá en Colombia, Buenos Aires en la Argentina, Lima en Perú, México DF en México, Quito en Ecuador y Santiago de Chile en Chile. Los hallazgos generales del estudio determinaron la prevalencia de hipertensión arterial que fue en promedio 18\% (9-29\%), la de hipercolesterolemia 14\% (6-20\%), la de diabetes 7\% (4-9\%), la de síndrome metabólico 20\% (14-27\%), la de obesidad 23\% (18-27\%) y la de tabaquismo 30\% (22-45\%). Alrededor del $13 \%$ de los sujetos refirieron que tenían miembros de la familia con enfermedad cardiovasculary el $2 \%$ manifestaron que habían sufrido un infarto o accidente vascular cerebral previos. ${ }^{2}$

Un estudio sobre los factores de riesgo cardiovascular realizado en nuestro país y publicado en 1998 encontró en 1606 sujetos entre 20 y 74 años de una población urbana una prevalencia de 6,5\% de diabetes mellitus $17,1 \%$ de hipertensión arterialy $31,6 \%$ de obesidad. ${ }^{3}$

Otro estudio sobre los factores de riesgo cardiovascular, realizado por la Sociedad Paraguaya de Cardiología junto con la Sociedad Interamericana de Cardiología entre los años 2003-2004 y publicado en el Congreso Sudamericano de Cardiología en Chile en el año 2005, indica que en el Paraguay la prevalencia del sedentarismo era 44\%, obesidad 21\%, hipertensión arterial 35\%, diabetes mellitus 8\% y tabaquismo $23 \%$. Estos datos afirmaban que el Paraguay era el país con más alta tasa de sedentarismo e hipertensión arterial en Sudamérica. ${ }^{3}$

Datos estadísticos del Ministerio de Salud Pública y Bienestar Social de Paraguay del año 2005 demostraban que la mortalidad por causa cardiovascular constituyó el 22\%; siendo ésta la de mayor cifra entre las denominadas enfermedades crónicas no transmisibles; es decir, 5.200 muertes fueron por enfermedades cardiovasculares, con un cálculo diario de 14 muertes por día. Análisis estimativos de proyección estimaban que para el año 2020 llegarían al 30\%, liderando siempre el primer lugar como causas de mortalidad entre las enfermedades crónicas no transmisibles. ${ }^{3}$

En el año 2011 se realizó en nuestro país la primera encuesta nacional de factores de riesgo cardiovascular y de enfermedades crónicas no transmisibles, según el método paso a paso de la Organización Mundial de la Salud y Organización Panamericana de la Salud. Se encuestaron a 2.750 personas en todo el país, con 1.650 en el área urbana y 1.100 en el área rural, en personas entre 15 y 75 años. Los principales hallazgos preliminares fueron: $74,5 \%$ de los encuestados no realizaban actividad física en su tiempo libre, es decir eran sedentarios; tabaquismo $14,1 \%$, sobrepeso $57,6 \%$, obesidad 22,8\%, diabetes mellitus 9,7 \% e hipertensión arterial $32,2 \%{ }^{4}$

En los países de ingresos bajos y medios como nuestro país se está observando un rápido incremento del número de jóvenes y adultos con sedentarismo, sobrepeso y obesidad, con las consecuencias que éstas desencadenan. La obesidad, junto con el sobrepeso, es el factor de riesgo cardiovascular más común en pacientes que han sufrido un infarto de miocardio. Más de dos tercios de los pacientes con enfermedad coronaria tienen sobrepeso u obesidad..$^{5-6}$ 
Esta investigación tiene la intención de ser un instrumento de utilidad y sirva de referencia para determinar los factores de riesgo cardiovascular en la población adulta joven del área urbana de la ciudad de Coronel Oviedo. Se utilizó el método progresivo paso a paso (steps) de vigilancia de factores de riesgo cardiovascular, de la Organización Mundial de la Salud y de la Organización Panamericana de la Salud. Este método consiste en un proceso secuencial, que incluye la recolección estandarizada de información, flexibilidad para incorporar factor de riesgo cardiovascular y diferentes niveles de medición. Aporta estimaciones validas, confiables y comparables de los factores de riesgo cardiovascular.

\section{Objetivo}

- Determinar la prevalencia de hipertensión arterial, diabetes mellitus, sobrepeso, obesidad y tabaquismo como factores de riesgo cardiovascular en una población del área urbana de la ciudad de Coronel Oviedo, Paraguay.

\section{Sujetos y métodos}

Diseño: estudio observacional, descriptivo de corte transversal.

Población de estudio: varones y mujeres entre 20 y 40 años de edad, domiciliados en el área urbana de Coronel Oviedo, entre los meses de junio a agosto del 2012.

Criterios de inclusión: pobladores permanentes de la ciudad

Criterios de exclusión: sujetos que se rehusaban a participar del estudio

Muestreo: aleatorio simple, por sorteo realizado en 10 barrios de la ciudad de Coronel Oviedo y donde se encuestó 15 viviendas por barrio.

Reclutamiento: voluntario, casa por casa, previo consentimiento informado sobre el estudio a realizar.

Variables: género, peso, estatura, índice de masa corporal, tabaquismo, hipertensión arterial y glicemia. Se catalogó a los individuos participantes como hipertensos cuando la presión arterial era $\geq 140 / 90 \mathrm{~mm} \mathrm{Hg}$ registrada en condiciones basales, en dos mediciones y en dos posiciones (sentado y en pie) utilizando esfigmomanómetros convencionales, de la marca ASPEN calibrados para este estudio, según las recomendaciones de la American Heart Association. ${ }^{12}$ Se incluyó a las personas diagnosticadas con hipertensión con tratamiento regular.

La glicemia basal se determinó con un ayuno mínimo de 8 horas y se utilizó como criterio diagnóstico las recomendaciones de American Diabetes Association ${ }^{13}$ estableciéndose un valor de glicemia en ayunas $>126$ $\mathrm{mg} / \mathrm{dL}$, realizado en el laboratorio, con el método automatizado y cinético. Se incluyeron además a los individuos diagnosticados con diabetes, con seguimiento médico y tratamiento regular.

Análisis de datos: las variables cuantitativas se expresan en medias $\pm D S$ y las cualitativas en porcentajes.

Cálculo del tamaño de la muestra: la proporción esperada de hipertensión arterial fue 32\% ${ }^{4}$. La amplitud del intervalo de confianza fue 0,16, el nivel de confianza fue 95\%. El tamaño de muestra mínimo fue 131 sujetos.

Cuestiones éticas: en todo momento se mantuvo la confidencialidad de los datos. Se respetó la autonomía 
de los sujetos de negarse a participar del estudio. No se discriminó por sexo, creencias religiosas ni políticas. El beneficio otorgado fue que los sujetos que fueron detectados con patologías eran remitidos al Hospital Regional Dr. José A. Samudio, Coronel Oviedo, para su tratamiento.

\section{Resultados}

Se incluyó a 139 sujetos. La edad media fue 28,8 años, la distribución según sexo fue $63 \%$ femenino y $37 \%$ masculino.

\section{GRÁFICO 1}

\section{Prevalencia de factores de riesgo cardiovascular en población joven (n 139)}

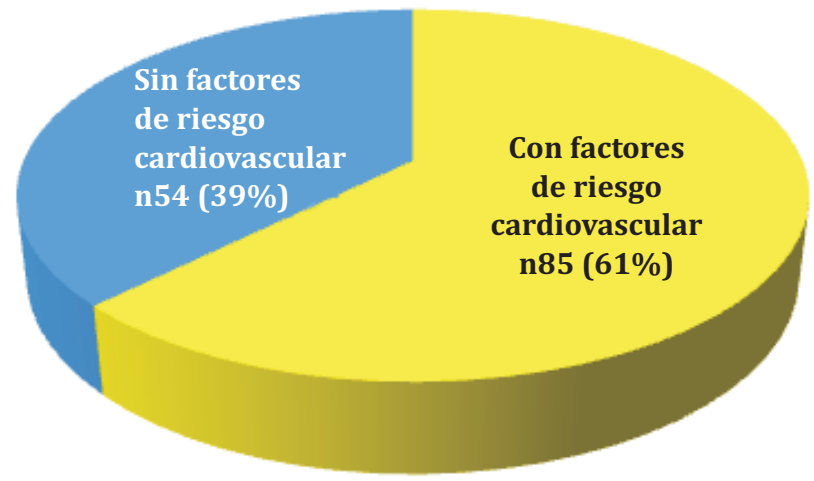

Los factores de riesgo cardiovascular más frecuentes fueron el sobrepeso, la hipertensión arterial y el tabaquismo (gráfico 2).

\section{GRÁFICO 2}

\section{Riesgos cardiovasculares en población joven (n 139)}

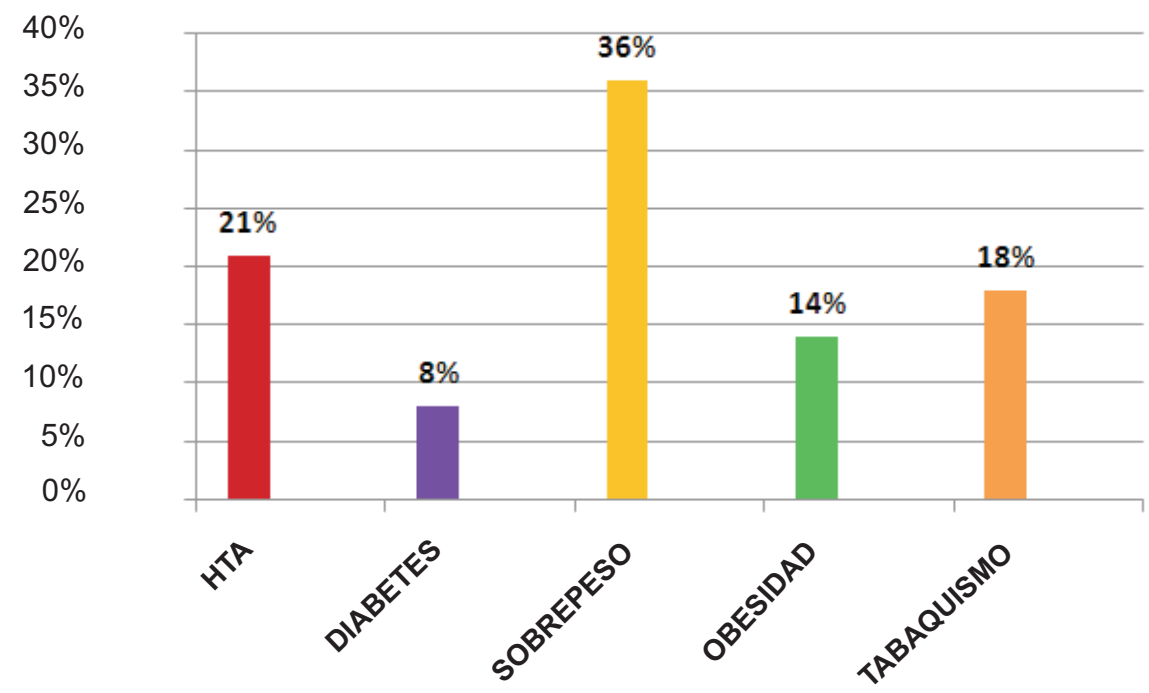

Reagrupando a la muestra según el género, en los varones predominó el tabaquismo y en las mujeres la obesidad (gráfico 3). 


\section{GRÁFICO 3}

\section{Distribución de factores de riesgo cardiovascular en relación al género (n 139)}

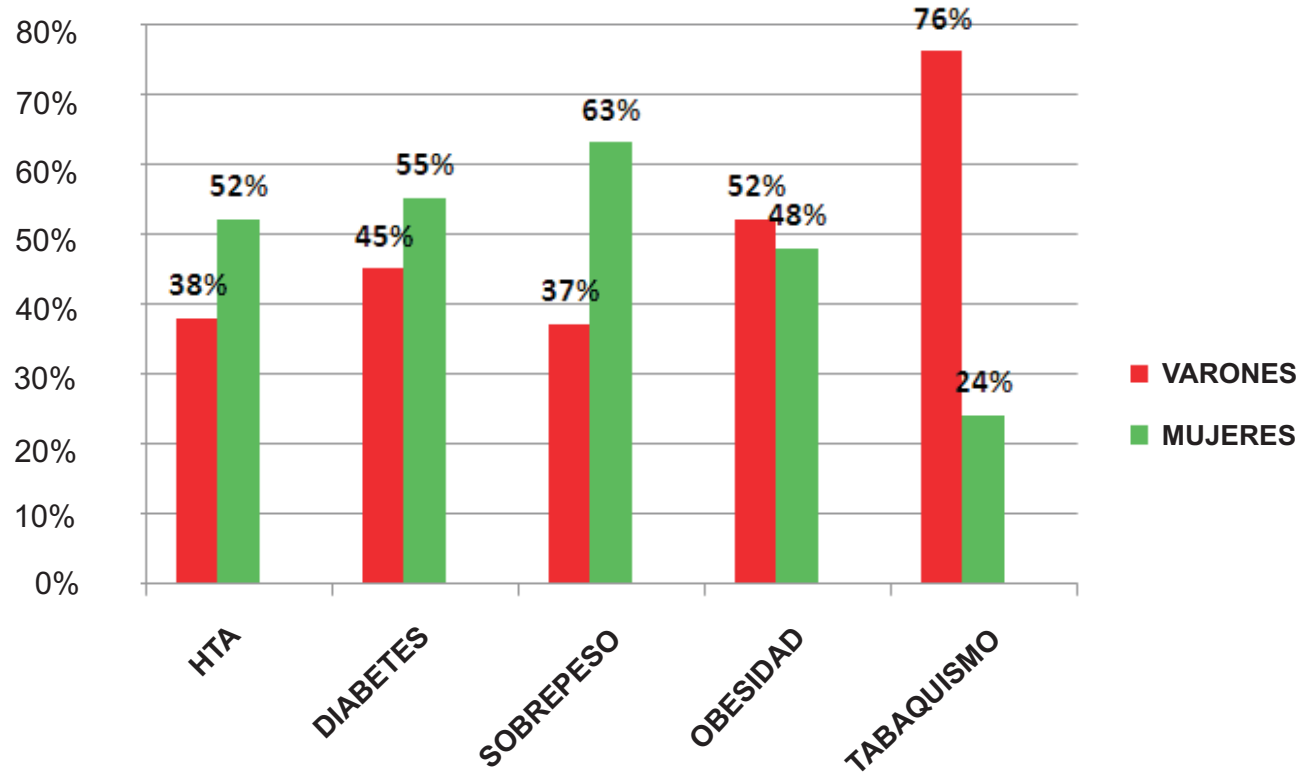

Reagrupando a los sujetos según la cantidad de factores de riesgo cardiovascular en cada uno de ellos, 19\% presentaba dos de ellos y 6\% tres de ellos (gráfico 4).

\section{GRÁFICO 4}

\section{Coexistencia de varios factores de riesgo (FR) cardiovascular en una población joven (n 139)}

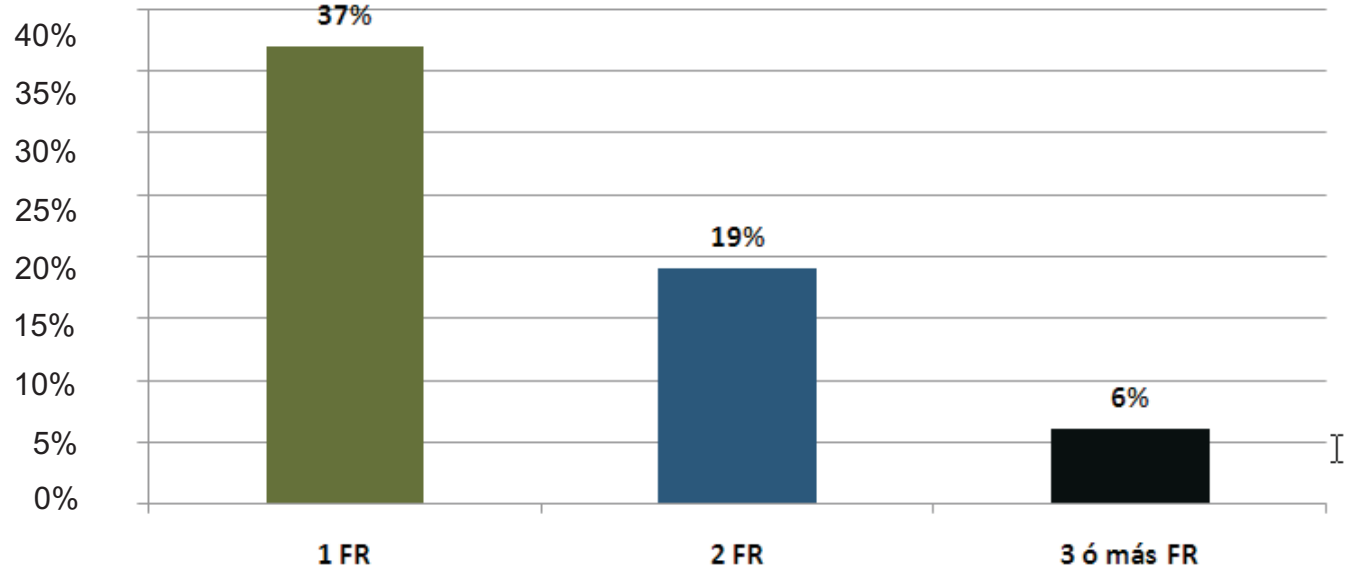

\section{Discusión}

El estudio ha determinado la frecuencia de varios de los factores de riesgo cardiovascular. El 23\% de los encuestados tenían hipertensión arterial, constituyendo un dato revelador, pues evidencia una frecuencia alta de esta condición clínica en esta franja etaria. La hipertensión arterial es un factor de riesgo cardiovascular muy prevalente en el mundo y especialmente abrumador en los países de bajos y medianos ingresos. Informes de la Organización Mundial de la Salud destacan la importancia de las enfermedades crónicas tales como la hipertensión, como obstáculo al logro de un buen estado de salud. ${ }^{7}$ 
La prevalencia de diabetes en la población estudiada (8\%) da la pauta del alto porcentaje de diabéticos en sujetos jóvenes en el área urbana de nuestra ciudad, comparada con la población general de nuestro país que según los últimos datos del año 2011 es de 9,7\%. La incidencia de la enfermedad cardiovascular en hombres y mujeres con diabetes mellitus tipo 2 es hasta 3 a 4 veces mayor que en individuos no afectados, es más, la diabetes mellitus está asociada con una tasa de mortalidad cardiovascular de más del 70\%, y las personas con diabetes mellitus tipo 2 tienen 2 a 3 veces mayor probabilidad de morir de enfermedad cardiovascular que la personas sin antecedente de diabetes mellitus.

El sobrepeso fue el factor de riesgo más predominante (36\%). Haciendo una comparación con la primera encuesta nacional de factores de riesgo hecha en nuestro país en el 2011 y haciendo la salvedad de la diferencia de la población estudiada en ese estudio fue de 15 - 75 años, en la misma la prevalencia de sobrepeso fue mucho mayor (57,6\%). Esto evidencia que ya existe un alto porcentaje de personas con sobrepeso en los jóvenes encuestados. En cuanto a la obesidad, una frecuencia de 15\%, comparable con los datos de la primera encuesta nacional de factores de riesgo cardiovascular del 2011 donde se halló una prevalencia de obesidad del $22,8 \%{ }^{4}$

Un estudio realizado en la Universidad Austral de Chile en el año 2007, con una muestra de 385 personas entre 17 - 26 años, determinó que 28,6\% de los estudiantes presentaba exceso de peso, de los cuales 23,4\% correspondió a sobrepeso y $5,2 \%$ a obesidad; sin diferencias significativas según género y quintil de ingreso. ${ }^{9}$

El 65\% de la población mundial vive en países donde el sobrepeso y la obesidad se cobran vidas; ambas son el quinto factor principal de riesgo de defunción en el mundo. Cada año fallecen por lo menos 2,8 millones de personas adultas como consecuencia del sobrepeso o la obesidad. Además, el 44\% de la carga de diabetes y el $23 \%$ de la carga de cardiopatías isquémicas son atribuibles al sobrepeso y la obesidad. ${ }^{10}$

El tabaquismo tuvo una frecuencia del 18\%, siendo esta cifra superior a la de la primera encuesta nacional, donde determinaron que ésta era del 14,1\%. ${ }^{4}$ Este hábito es la principal causa de muerte prematura evitable en el mundo. A causa del tabaco mueren 5,4 millones de personas por año y murieron 100 millones de personas en el siglo XX. Si continúa la tendencia actual, aumentaran las muertes en este siglo debido al consumo de tabaco. ${ }^{11}$

En nuestro estudio la diabetes mellitus, la hipertensión arterial y la obesidad son más frecuentes en el sexo femenino, coincidentes con otro estudio realizado en nuestro país. ${ }^{3}$

Los datos obtenidos permiten evaluar, en un contexto global, las altas cifras de portadores de los diferentes factores de riesgo cardiovascular, $y$, en forma general, comparables con las cifras determinadas en los diferentes estudios anteriormente realizados, a nivel nacional. ${ }^{3,4}$

Se tendrá que realizar otros estudios a futuro, siguiendo la misma metodología, a fin de ir monitoreando la evolución de los distintos factores de riesgo detectados en esta investigación. Se debería implementar estrategias dirigidas a toda la población, como la promoción de un régimen alimentario saludable acompañado de la actividad física. El tratamiento de factores de riesgo tales como la hipertensión y la diabetes mellitus requiere de intervenciones oportunas, incluso el aumento de los impuestos al tabaco, y la elaboración de leyes como medidas para disminuir el tabaquismo.

Concluyendo, este estudio en jóvenes de Coronel Oviedo encontró que 21\% padece hipertensión arterial, $8 \%$ diabetes mellitus, 36\% sobrepeso, 14\% obesidad y 18\% tabaquismo. Hay mayor tendencia de 
padecer hipertensión arterial, diabetes y obesidad en el sexo femenino. Deberían realizarse investigaciones poblaciones a mayor escala dada la elevada e inusual prevalencia de hipertensión arterial detectada en este estudio.

\section{Referencias bibliográficas}

1. Organización Mundial de la Salud. Enfermedades no transmisibles y factores de riesgo cardiovascular. Boletín Oficial 14 septiembre 2011. Disponible en: http://www.who.int/mediacentre/news/releases/2011/NCDs_pro files_20110914/es/

2. Hernández-Hernández R, Silva H, Velasco M, Pellegrini F, Macchia A, Escobedo J, Vinueza R, Schargrodsky H, Champagne B, Pramparo P, Wilson E; CARMELA Study Investigators. Hypertension in seven Latin American cities: the Cardiovascular Risk Factor Multiple Evaluation in Latin America (CARMELA) study. J Hypertens. 2010 Jan; 28(1): 24-34.

3. Jiménez JT, Palacios C de, Cañete F, Barriocanal LA, Medina U, Figueredo R, Melgarejo MV de, Martínez S, Weik S, Kiefer R, Alberti KGMM, Moreno Azorero R. Prevalence of diabetes mellitus and associated cardiovascular risk factors in an adult urban population in Paraguay. Diabetic Medicine 1998; 15: 334-338

4. Paraguay. Ministerio de Salud Pública y Bienestar Social. Primera Encuesta Nacional de Factores de Riesgo Cardiovascular y Enfermedades Crónicas no Transmisibles. MSPBS; 2011.

5. Romero-Corral A, Montori VM, Somers VK, Korinek J, Thomas RJ, Allison TG, et al. Association of bodyweight with total mortality and with cardiovascular events in coronary artery disease: a systematic review of cohort studies. Lancet. 2006; 368: 666-78.

6. Lopez-Jiménez F, Wu CO, Tian X, O'Connor C, Rich MW, Burg MM, et al. Weight change after myocardial infarction-the Enhancing Recovery in Coronary Heart Disease patients (ENRICHD) experience. Am Heart J. 2008; 155: 478-84.

7. WHO. The World Health Report, 2002: reducing risks, promoting healthy life. Geneva: World Health Organization; 2003. Report of the Commission Macroeconomics and Health: investing in health for economic development. Geneva:WHO. 2001.

8. Booth GL, Kapral MK, Fung K, Tu JV. Relation between age and cardiovascular disease in men and women with diabetes compared with non diabetic people: a population-based retrospective cohort study. Lancet 2006; 368 : 29-36.

9. Martínez S MA, Leiva O AM, Sotomayor C C, Victoriano R T, Von Chrismar P AM, Pineda B S. Cardiovascular risk factors among university students. Rev Med Chil. 2012 Apr; 140(4): 426-35.

10. Organización Mundial de la Salud. Sobrepeso y Obesidad. Boletín de prensa; nota descriptiva 311. Mayo 2012.

11. WHO Report on the Global Tobacco Epidemic 2008: The MPOWER Packaged. Geneva: World Health Organization; 2008.

12. Chobanian AV, Bakris GL, Black HR, Cushman WC, Green LA, Izzo JL Jr et al. Seventh report of the Joint National Committee on Prevention, Detection, Evaluation, and Treatment of High Blood Pressure. Hypertension. 2003 Dec; 42(6): 1206-52.

13. American Diabetes Association. Standards of medical care in diabetes 2013. Diabetes Care. 2013 Jan; 36 Suppl 1: S11-66. 